\title{
Influência do fumo na atividade da amilase salivar e na curva glicêmica
}

\author{
Influence of smoking on salivary amylase \\ activity and glycemic curve
}

Patricia MORIEL ${ }^{1}$

Hermes Lima MADUREIRA²

Áurea Kátia Yuuko UWAGOYA²

Luana WLIAN²

Eder de Carvalho PINCINATO3

RE S U M O

\section{Objetivo}

Determinar a atividade da amilase salivar e a relação com a glicemia, antes e após a ingestão de carboidratos em fumantes e não fumantes, uma vez que in vitro a exposição da saliva à fumaça do cigarro induz à redução da atividade da amilase salivar e poderia influenciar na absorção dos carboidratos da dieta.

\section{Métodos}

Foram avaliados voluntários fumantes $(n=10)$ e não fumantes $(n=10)$. Realizou-se coletas da saliva antes e após o fumo e determinou-se a glicemia antes e após a ingestão de $72 \mathrm{~g}$ de carboidratos. Para glicemia usaram-se tempos de 0,15, 30, 60, 120 minutos. A determinação da atividade da amilase salivar foi realizada por meio de kits comerciais. A glicemia foi determinada utilizando o aparelho Glicomiter (Accu-Chek-Roche). As análises estatísticas foram realizadas no software Sigmastat, utilizou-se o método Teste $t$ pareado $(p<0,05)$.

\section{Resultados}

O aumento da glicemia aos 15, 30, 60 e 90 minutos foi de 3,9; 11,9; 34,8 e 22,7\% para os não fumantes e 4,$9 ; 6,5 ; 13,8$ e $9,7 \%$ para os fumantes, respectivamente. No pico máximo de absorção tem-se uma glicemia de $21,0 \%$ maior nos pacientes não fumantes. A atividade da amilase salivar antes e após alimentação apresentou-se 75,0\% menor nos indivíduos fumantes.

\section{Conclusão}

Estes resultados sugerem que o fumo inibe a amilase e influencia na diminuição da digestão/absorção de carboidratos, consequentemente na concentração de glicose sanguínea, reduzindo assim o aporte energético ingerido.

Termos de indexação: Alfa-amilases salivares. Glicemia. Processos do sistema digestório. Tabaco.

\footnotetext{
1 Universidade Estadual de Campinas, Faculdade de Ciências Médicas, Departamento de Patologia Clínica. R. Tessália Vieira de Camargo, 126, 13081-970, Campinas, SP, Brasil. Correspondência para/Correspondence to: P. MORIEL. E-mail: <morielpa@fcm.unicamp.br>.

2 Universidade Camilo Castelo Branco. São Paulo, SP, Brasil.

${ }^{3}$ Universidade Presbiteriana Mackenzie. São Paulo, SP, Brasil.
} 


\section{A B S T R A C T}

\section{Objective}

The objective of this study was to determine salivary amylase activity and its relationship with glycemia before and after smokers and nonsmokers ingested carbohydrates. Since cigarette smoke reduces salivary amylase activity in vitro, it may affect dietary carbohydrate absorption.

\section{Methods}

Twenty volunteers participated in this study, 10 smokers and 10 nonsmokers. Samples of saliva were collected before and after the smokers had a cigarette and glycemia was determined before and after the ingestion of $72 \mathrm{~g}$ of carbohydrates. Glycemia was measured 0, 15, 30, 60 and 120 minutes after carbohydrate intake. Salivary amylase activity was determined by commercial kits. Glycemia was determined by a glucometer (Accu-Chek-Roche). The paired t-test was used for the statistical analyses, done by the software Sigmastat, with $p<0.05$.

\section{Results}

Glycemia 15, 30, 60 and 90 minutes after carbohydrate intake rose 3.9\%, 11.9\%, 34.8\% and 22.7\% in nonsmokers and $4.9 \%, 6.5 \%, 13.8 \%$ and $9.7 \%$ in smokers, respectively. The peak glucose absorption in nonsmokers was $21.0 \%$ greater than in smokers. Salivary amylase activity before and after eating was $75.0 \%$ smaller in smokers.

\section{Conclusion}

These results suggest that smoking inhibits amylase and has a negative impact on the digestion/absorption of carbohydrates, consequently in blood glucose levels, thereby reducing the amount of energy absorbed.

Indexing terms: Salivate alpha-amylases. Blood glucose. Digestive system bodisses. Tabaco.

\section{N T R O D U ÇÃ O}

Os alimentos dos quais o organismo depende não podem ser absorvidos, em suas formas naturais, através da mucosa gastrintestinal, se não forem submetidos a uma digestão preliminar, com exceção das vitaminas e sais minerais.

A cavidade bucal contém saliva, que é secretada por três pares de glândulas salivares: parótida, submandibular e sublingual. A saliva é constituída de aproximadamente $99,5 \%$ de água e age como um lubrificante para a mastigação na cavidade oral e deglutição. $\mathrm{O}$ pH da saliva situa-se ligeiramente para o lado ácido, cerca de 6,8, embora possa variar para um ou outro lado da neutralidade ${ }^{1}$.

Em condições basais, ocorre secreção de cerca de meio mililitro de saliva por minuto, quase totalmente do tipo mucoso, exceto durante o sono, quando a secreção se torna muito pequena. A secreção salivar é fator importante na manutenção da saúde bucal, com função de limpeza e proteção, por meio dos vários mecanismos fi- siológicos e mecânicos. Estes mecanismos ocorrem em resposta a estímulos táteis e gustativos na boca, assim como visual-olfatório e estimulações de centros cerebrais superiores².

O processo digestivo dos carboidratos tem início com a mastigação, que facilita o acesso das enzimas ao amido. A saliva contém a enzima alfa-amilase, conhecida antigamente como ptialina, cuja função é iniciar na boca a digestão do amido. Essa enzima catalisa a hidrólise das ligações internas $\alpha-1,4$ do amido, mas não consegue hidrolisar as ligações ramificadas $\alpha-1,6$. A $\alpha$-amilase secretada pelo pâncreas possui a mesma especificidade e a sua atividade enzimática total é consideravelmente maior que aquela da amilase salivar. A ação da $\alpha$-amilase salivar continua até que o alimento no estômago esteja misturado com o ácido gástrico, que inativa a enzima. Depois que a $\alpha$-amilase salivar é inativada pelo ácido gástrico com o pH em torno de 4,0, não haverá no estômago nenhum processamento adicional dos carboidratos. Dez minutos após penetrar no duodeno, o amido é quase comple- 
tamente transformado em maltose, maltotriose, maltooligossacarídios com ligações $\alpha-1,4$.

A saliva é o primeiro fluído corporal a entrar em contato com a fumaça inalada do cigarro, que é danosa à cavidade oral e está associada a muitas doenças, como por exemplo, o câncer oral. O tabaco é uma planta da família das Solanáceas da qual existem diversas variedades, sendo a principal a Nicotiana tabacum L. Seu uso surgiu aproximadamente no ano 1000 a.C., nas sociedades indígenas da América Central, em rituais mágicos-religiosos, pois acreditavam que o uso conteria o poder de predizer o futuro ${ }^{3}$.

A planta chegou ao Brasil, provavelmente, pela migração de tribos tupis-guaranis. Dependendo da região do cultivo, do solo, da secagem e do armazenamento, a folha do tabaco pode conter cerca de quinhentos constituintes químicos e, quando queimados, já foram isolados cerca de 4720 substâncias. O principal princípio ativo é a nicotina, um alcalóide que foi isolado em seu estado puro em 1828 por Ponsselt e Reiman. O tabagismo é hoje um dos principais problemas de saúde pública. Embora alguns aspectos científicos possam ser estudados, a relação causa e efeito entre o tabaco e várias moléstias já está plenamente demonstrada. É notória a participação do tabaco no aumento e/ou agravamento de doenças cardiovasculares, pulmonares, circulatórias, gastrintestinais, em numerosos tipos de cânceres, bem como em efeitos nocivos no feto, contribuindo para o incremento da morbidade e mortalidade populacional ${ }^{4}$.

A fumaça do cigarro é resultante da combustão incompleta do tabaco ${ }^{4}$. A composição real da fumaça liberada pelo fumante não depende apenas da composição do tabaco, como também da compactação do produto, do comprimento do cigarro, das características do filtro, do papel e da temperatura na qual o tabaco é queimado ${ }^{5}$.

Os principais efeitos da nicotina no sistema nervoso central são: elevação leve no humor (estimulação) e diminuição do apetite. A nicotina é considerada um estimulante leve, apesar de um grande número de fumantes relatarem que se sentem relaxados quando fumam. Essa sensação de relaxamento é provocada pela diminuição do tônus muscular. No sistema digestório provoca diminuição da contração do estômago, dificultando a digestão. Há um aumento da vasoconstrição e na força de contração cardíaca ${ }^{3}$. A probabilidade do aparecimento dessas complicações tem relação direta com o tempo de dependência, aumentando proporcionalmente com o grau de exposição ${ }^{5}$.

A síndrome de abstinência e a tolerância são alguns dos sinais que caracterizam o quadro de dependência provocado pelo uso de tabaco ${ }^{3}$. A dependência existe quando o indivíduo é dominado por uma vontade incontrolável de obter a droga. Sem ela, a ansiedade aumenta e a pessoa normalmente tem uma forte sensação de mal-estar. Quando a dependência existe, o organismo se encontra de tal maneira adaptado à droga que passa a necessitar dela de forma compulsiva. Neste caso, a retirada da droga provoca a chamada "síndrome de abstinência", uma situação na qual a pessoa, independentemente de sua vontade, sofre alterações no funcionamento do organismo, sendo que estas alterações às são graves ${ }^{6}$.

Diversos autores admitem que a dificuldade para o fumante abandonar o hábito de fumar se deve aos efeitos experimentados pela abstinência, buscando o alívio de um estado desagradável ${ }^{4,6}$. Além da fissura pelo tabaco, em humanos, a síndrome de abstinência ao cigarro inclui: bradicardia, desconforto gastrintestinal, aumento do apetite, ganho de peso, dificuldade de concentração, ansiedade, disforia, depressão e insônia 5 .

É bem conhecida a influência do fumo na atividade de algumas enzimas, como por exemplo, da elastase, que se encontra mais ativada em fumantes, aumentando a destruição da elastina e propiciando o enfisema pulmonar. A atividade das enzimas hepáticas também se altera pelo tabaco, promovendo a diminuição da vida média de algumas drogas? 
Nos últimos anos, estudos têm sido realizados para averiguar a relação entre parar de fumar e o ganho de peso. Muitos deles não acham diferença entre parar de fumar e o ganho de peso ${ }^{8-10}$; contudo outros estudos têm demonstrado um aumento real no peso após a retirada do taba$\mathrm{co}^{11-14}$. Muitos ex-fumantes relatam que, após parar de fumar, existe um ganho de peso corporal, mesmo mantendo a qualidade e quantidade alimentar. Não se sabe ainda o mecanismo bioquímico envolvido. Portanto o objetivo deste trabalho foi determinar a atividade da amilase salivar e a relação com a glicemia, antes e após a ingestão de carboidratos em fumantes e não fumantes, a fim de observar a influência do fumo na atividade da enzima e na digestão dos carboidratos.

\section{MÉ T O D O S}

Foram selecionados voluntários fumantes há pelo menos quatro anos ( $n=10)$, os quais fumam de dez a vinte cigarros por dia, e não fumantes $(n=10)$. Os voluntários foram previamente informados sobre a utilização do material biológico para a realização do trabalho, assinaram o Termo de Consentimento Livre Esclarecido e participaram como voluntários. O estudo seguiu os aspectos éticos recomendados pela Resolução 196/96 ${ }^{15}$ sobre Pesquisa Envolvendo Seres Humanos, bem como os princípios éticos. O projeto foi aprovado pelo Comitê de Ética da Universidade Camilo Castelo Branco sob o protocolo $n^{\circ}$ 634-847/04

\section{Coleta}

Foram realizadas coletas da saliva antes e depois da ingestão dos carboidratos. Para os voluntários fumantes a coleta da saliva foi realizada antes do fumo, após o fumo e após a ingestão dos carboidratos.

A glicemia foi determinada nos pacientes em jejum e nos tempos 15, 30, 60, 120 minutos após a ingestão de $200 \mathrm{~g}$ de batata-frita; este alimento foi o escolhido devido à maior aceitação pelos voluntários e foi ingerido em no máximo cinco minutos ( $200 \mathrm{~g}$ de batata frita correspondem a $72 \mathrm{~g}$ de carboidratos) ${ }^{16}$.

\section{Determinações analíticas}

A determinação da atividade da amilase salivar (UA/dL) foi realizada por meio de kits colorimétricos comerciais (Laborlab; sensibilidade metodológica: $8 \mathrm{UA} / \mathrm{dL}$; erro do método: $<9,3 \%$ ), utilizando diluição 1:700. A saliva foi centrifugada por vinte minutos a 3000rpm. Em seguida, foi retirado o sobrenadante e realizada as diluições sucessivas. A determinação da glicemia ( $\mathrm{mg} / \mathrm{dL}$ ) foi feita por método da química seca (glicose desidrogenase) utilizando o aparelho Accu-Chek Advantage (Roche; sensibilidade metodológica: $10 \mathrm{mg} / \mathrm{dL}$; erro do método: $<3,0 \%$ ).

Os valores foram representados como média e desvio-padrão. Os gráficos foram feitos no software Microsoft Excel. As análises estatísticas foram realizadas após tratamento dos dados no software Sigmastat, utilizou-se o método teste $t$ pareado $(p<0,05)$.

\section{RESULTADOSE DISCUSSÃO}

Nos voluntários fumantes foi realizada uma determinação também em jejum após o fumo, comparando-se, assim, a atividade da amilase salivar dos fumantes antes e após o fumo no jejum, observando que não existiram diferenças significativas (Figura 1).

Os resultados acima demonstram que, nos voluntários não fumantes, a atividade da amilase salivar antes da alimentação é menor; e quando o voluntário ingere o alimento esta atividade aumenta. Isto ocorre, devido à maior secreção de saliva na cavidade bucal, durante a mastigação.

Nos voluntários fumantes, observa-se que a atividade da amilase salivar é bem menor em todas as coletas em relação aos não fumantes, 
ocorrendo um pequeno aumento depois da alimentação. Estes resultados estão de acordo com Nagler et al. ${ }^{16,17}$, os quais demonstraram, in vitro, a exposição da saliva a nove puffs de fumaça do cigarro induz à elevação de proteínas carboniladas, provavelmente devido à reação de seus grupos tióis com os aldeídos presentes na fase gasosa, causando uma redução de 34\% da atividade da amilase salivar. Goi et al. ${ }^{18}$ também demonstram a diminuição da atividade da amilase salivar em fumantes e das enzimas antioxidantes. Os resultados obtidos no gráfico acima mostram que a atividade da enzima antes e após alimentação apresentou-se 87,3\% e 87,5\% menor nos indivíduos fumantes. Além disso, Zappacosta et al. ${ }^{19,20}$ verificaram também que um cigarro é suficiente para diminuir a atividade enzimática em $40 \%$. Segundo Moldeus et al. ${ }^{21}$ e Joshi et al. ${ }^{22}$, os aldeídos do tabaco inibem diretamente a amilase salivar, e que esta atividade, na presença da glutationa, um antioxidante natural presente na saliva, é preservada.

Em estudos com voluntários fumantes, mediu-se a atividade enzimática e a concentração da Glutationa (GSH) na saliva antes e depois de fumar um único cigarro. Os estudos demonstraram que um cigarro é suficiente para diminuir a atividade enzimática, assim como ficou evidente o efeito protetor exercido pela glutationa salivar cuja concentração ficou significantemente redu-

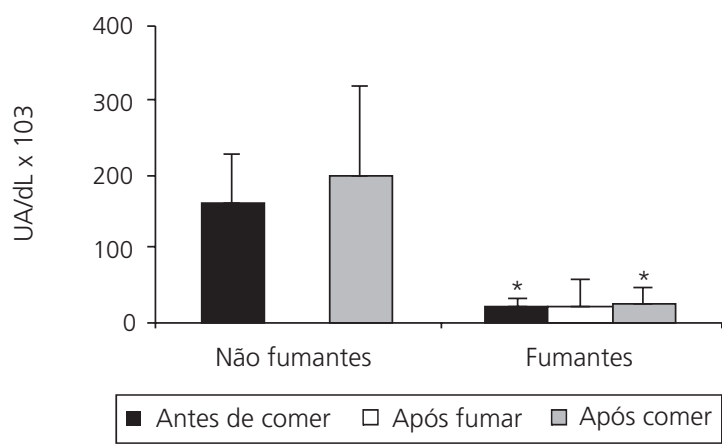

Figura 1. Atividade da amilase salivar em fumantes e não fumantes. São Paulo (SP), 2006.

Nota: * significante em relação aos não fumantes. zida $^{19,20}$. A glutationa reage com a acroleína e o crotonaldeído, os mais importantes aldeídos insaturados ${ }^{23}$.

Em ratos e coelhos verificou-se que a fase condensada da fumaça também diminui a glutationa (cerca de $50 \%$ ) sem nenhum aumento na glutationa oxidada. A toxicidade da fase condensada sobre os hepatócitos e células pulmonares de ratos é decorrente da depleção da glutationa reduzida e, consequentemente, perda da integridade da membrana celular. O consumo da glutationa deve ser pela sua conjugação com componentes eletrofílicos do cigarro. Compostos tióis, como N-acetilcisteína na concentração de $1 \mathrm{mM}$, tornam-se protetores pela liberação direta das sulfidrilas 21,22 .

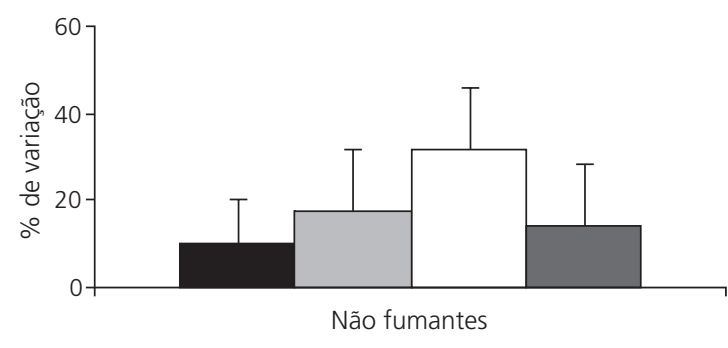

15 minutos $\square 30$ minutos $\square 60$ minutos* $\square 120$ minutos

Figura 2. Porcentagem de variação da glicemia nos voluntários não fumantes após a ingestão de $72 \mathrm{~g}$ de carboidrato. São Paulo (SP), 2004.

Nota:* significante em relação ao tempo zero.

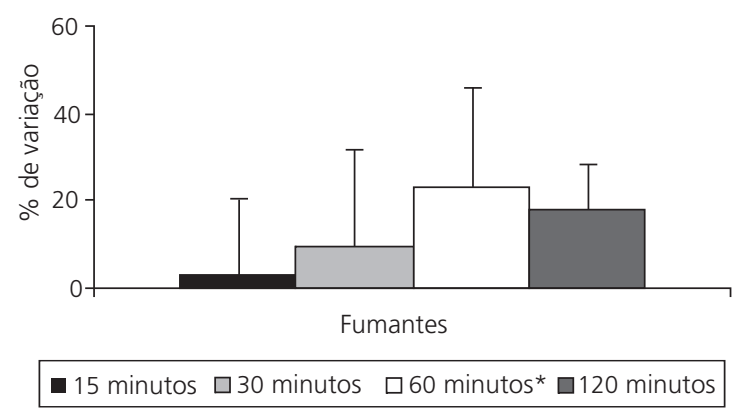

Figura 3. Porcentagem de variação da glicemia nos voluntários fumantes após a ingestão de $72 \mathrm{~g}$ de carboidrato. São Paulo (SP), 2004.

Nota: * significante em relação ao tempo zero. 
A interação entre os aldeídos do cigarro e os grupos tióis presentes na amilase salivar e na glutationa (antioxidante natural) é, provavelmente, responsável pela redução da atividade enzimática em fumantes, de forma direta, inibindo a enzima, e indireta, através da inativação da glutationa ${ }^{21,22}$.

Os valores obtidos da glicemia nos voluntários não fumantes antes, 15, 30, 60 e 120 minutos após a ingestão foram de Média $(M)=88,7$, Desvio-Padrão (DP)=4,7mg/dL; $M=96,9, D P=7,6 \mathrm{mg} /$ $\mathrm{dL} ; \mathrm{M}=103,4, \mathrm{DP}=10,0 \mathrm{mg} / \mathrm{dL} ; \mathrm{M}=117,3, \mathrm{Dp}=12,6 \mathrm{mg} /$ $\mathrm{dL}$ e $\mathrm{M}=102,3, \mathrm{DP}=16,1 \mathrm{mg} / \mathrm{dL}$, respectivamente (Figura 2).

Os valores obtidos da glicemia nos voluntários fumantes antes, 15, 30, 60 e 120 minutos após a ingestão foram de $M=91,8, D P=12,7 \mathrm{mg} / \mathrm{dL}$; $M=94,7, D P=15,0 \mathrm{mg} / \mathrm{dL} ; M=99,4, D P=15,9 \mathrm{mg} / \mathrm{dL} ;$ $M=110,5, D P=24,5 \mathrm{mg} / \mathrm{dL}$ e $M=107,1, D P=11,5 \mathrm{mg} /$ $\mathrm{dL}$, respectivamente (Figura 3 ).

Em relação à glicemia, observa-se que a porcentagem de aumento da glicose sanguínea nos tempos 15, 30, 60 e 120 minutos foi de 9,6; 17,$0 ; 32,7$ e 15,1\% para os não fumantes, enquanto que, nos voluntários fumantes, a porcentagem de aumento foi de 3,5; 10,0; 21,7 e 17,8\%, respectivamente. Além disso, observa-se que no tempo sessenta minutos é o pico máximo de absorção da glicose, e apresenta-se uma glicemia

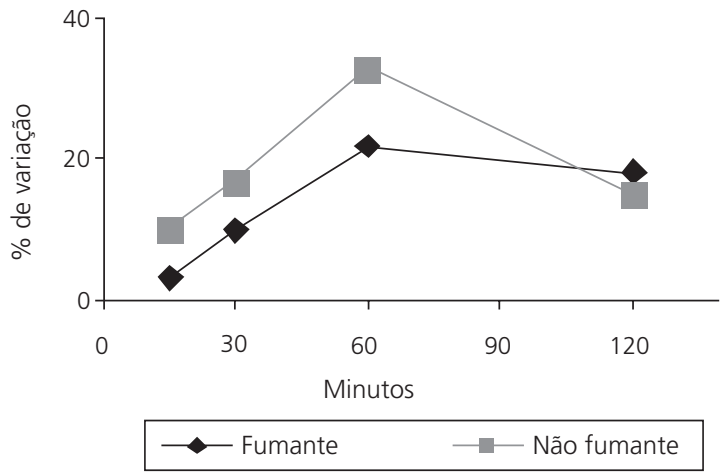

Figura 4. Comparação da porcentagem de variação da glicemia de fumantes e não fumantes nos diferentes tempos. São Paulo (SP), 2004

Nota: não existiram diferenças significativas. de 11,0\% maior para os voluntários não fumantes, em relação aos fumantes. Portanto, esta tendência sugere uma diminuição da absorção dos carboidratos ingeridos (Figura 4).

Nos últimos anos, estudos têm sido realizados para averiguar a relação de parar de fumar e ganhar de peso. Muitos deles não acham correlação significativa entre os dois fatos ${ }^{8-10}$. Contudo, outros estudos têm demonstrado um aumento real no peso após a parada de fumar ${ }^{11-14}$. Alguns mecanismos são propostos para este aumento de peso, como o aumento da atividade da lipase lipoproteíca do tecido adiposo, fazendo um maior depósito de ácidos graxos neste tecido ${ }^{19}$, o aumento do aporte calórico, diminuição da taxa metabólica e diminuição da atividade física ${ }^{20}$. Estes trabalhos vão de encontro com os resultados aqui demonstrados, onde se sugere que o cigarro diminuiu a absorção de carboidratos.

Também é interessante a observação da curva glicêmica dos fumantes. Ela é similar à dos não fumantes, porém, no tempo 120 minutos, a glicemia dos não fumantes já está praticamente voltando às concentrações iniciais, enquanto que a dos fumantes tem uma tendência a permanecer elevada. Isto poderia sugerir que ainda está ocorrendo a absorção da glicose, pela maior lentidão da digestão destes indivíduos, devido à diminuição da atividade da amilase salivar ou diminuição da entrada da glicose nos tecidos. Alguns trabalhos demonstram que existe uma resposta metabólica menor destes indivíduos frente à elevação da glicemia ou por uma diminuição de produção de insulina e/ou por um aumento da resistência das células ${ }^{24-28}$.

\section{CONCLUSÃO}

Estes resultados sugerem que o fumo inibe a atividade da amilase salivar e influencia na diminuição da digestão/absorção de carboidratos, consequentemente na concentração de glicose sanguínea, diminuindo assim o aporte energético ingerido. 
Este trabalho ainda sugere que o aumento do ganho de peso após a parada de fumar poderia ser devido à menor digestão/absorção dos carboidratos que ocorre por causa da inibição da amilase, mesmo com a mesma quantidade/qualidade da dieta nestas pessoas.

\section{COLABORADORES}

P. MORIEL participou da orientação do projeto e da redação do artigo. H.L. MADUREIRA responsável pelos experimentos com cinética enzimática. A.K.Y. UWAGOYA e L. WILIAN responsáveis pelos recrutamento, agendamento e curva glicêmica dos pacientes. E.C. PINCINATO responsável pelas análises realizadas e participou da redação do artigo.

\section{REFERÊ NCIAS}

1. Pedersen AM, Bardow A, Beier JS, Nauntofte B. Saliva and gastrointestinal functions of taste, mastication, swallowing and digestion. Oral Dis. 2002; 8(3):117-29.

2. Nieuw AV, Ligtenberg AJ, Veerman EC. Implications for diagnostics in the biochemistry and physiology of saliva. Ann N Y Acad Sci. 2007; 1098(1):1-6.

3. Centro Brasileiro de Informação sobre Drogas Psicotrópicas. São Paulo: Unifesp; 2001.

4. Oga S. Fundamentos de toxicologia. São Paulo: Atheneu; 1996.

5. O'Brien CP. Drug addiction and abuse. In: Hardman JG, Limbird LE, editors. Goodman and Gilman's the pharmacological basis of therapeutics. $10^{\text {th }} \mathrm{ed}$. New York: Pregamon; 2001. p.621-42.

6. Planeta CS, Cruz FC. Bases neurofisiológicas da dependência de tabaco. Rev Psiquiatr Clin. 2005; 32(5):251-8.

7. Rosemberg J. Tabagismo: sério problema de saúde pública. São Paulo: Artmed; 1981.

8. Ferrara CM, Kumar M, Nicklas B, McCrone S, Goldberg AP. Weight gain and adipose tissue metabolism after smoking cessation in women. Int J Obes Relat Metab Disord. 2001; 25(9):1322-6.

9. Filozof C, Fernandez PMC, Fernandez CA. Smoking cessation and weight gain. Obes Rev. 2004; 5(2):95-103.

10. Allen SS, Allen AM, Mooney M, Bade T. Short-term weight gain by menstrual phase following smoking cessation in women. Eat Behav. 2009; 10(1):52-5.
11. Burke JP, Hazuda HP, Stern MP. Rising trend in obesity in Mexican Americans and non-Hispanic whites: is it due to cigarette smoking cessation? Int J Obes Relat Metab Disord. 2000; 24(12): 1689-94.

12. Gruber J, Frakes M. Does falling smoking lead to rising obesity. J Health Econ. 2006; 25(2):183-97.

13. Levine MD, Marcus MD, Perkins KA. Women, weight, and smoking: a cognitive behavioral approach to women's concerns about weight gain following smoking cessation. Cogn Behav Pract. 2003; 10(2):105-11.

14. Chaney SE, Sheriff S. Weight gain among women during smoking cessation: testing the effects of a multifaceted program. AAOHN J. 2008; 56(3):99-105.

15. Nagler RM, Klein I, Reznick AZ. The interaction between saliva and cigarette smoke and its devastating biological effects as related to oral cancer. Harefuah. 2001; 140(7):614-77.

16. Sociedade Brasileira de Diabetes [Internet]. São Paulo: Sociedade Brasileira de Diabetes [acesso 2008 jun 10]. Disponível em: <www.diabetes. org.br/apundendo/contagem_carboidratos/tabela decontagem.php $>$.

17. Nagler R, Lischinsky S, Diamond E, Drigues N, Klein I, Reznick AZ. Effect of cigarette smoke on salivary proteins and enzyme activities. Arch Biochem Biophys. 2000; 379(2):229-36.

18. Goi N, Hirai Y, Harada H, Ikari A, Ono T, Kinae N, et al. Comparison of peroxidase response to mental arithmetic stress in saliva of smokers and non-smokers. J Toxicol Sci. 2007; 32(2):121-7.

19. Zappacosta B, Persichilli S, De Sole P, Mordente A, Giardina B. Effect of smoking one cigarette on antioxidant metabolites in the saliva of healthy smokers. Arch Oral Biol. 1999; 44(6):485-8.

20. Zappacosta B, Persichilli S, Mordente A, Minucci A, Lazzaro D, Meucci E, et al. Inhibition of salivary enzymes by cigarette smoke and the protective role of glutatione. Hum Exp Toxicol. 2002; 21(1):7-11.

21. Moldeus P, Berggren M, Grafstrom R. N-acetylcysteine protection against the toxicity of cigarette smoke and cigarette smoke condensates in various tissues and cells in vitro. Eur J Respir Dis Suppl. 1985; 139(1):123-9.

22. Joshi UM, Kodavanti PR, Mehendale HM. Glutathione metabolism and utilization of external thiols by cigarette smoke-challenged, isolated rat and rabbit lungs. Toxicol Appl Pharmacol. 1988; 96(2): 324-35.

23. Reddy S, Finkelstein El, Wong PS, Phung A, Cross $C E$, Vander Vliet $A$. Identification of glutathione 
modifications by cigarette smoke. Free Radic Biol Med. 2002; 33(11):1490-8.

24. Eliasson B. Cigarette smoking and diabetes. Prog Cardiovasv Dis. 2003; 45(5):405-13.

25. Perry IJ, Wannamethee SG, Walker MK, Thomson AG, Whincup PH, Shaper AG. Prospective study of risk factors for development of nominsulin dependent diabetes in middle aged British men. BMJ. 1995; 310(6979):560-4.

26. Wannamethee SG, Shaper AG, Perry IJ. Smoking as a modifiable risk factor for type 2 diabetes in middleaged men. Diabetes Care. 2001; 24(9): 1590-5.
27. Uchimoto S, Tsumura K, Hayashi T, Suematsu C, Endo G, Fujii $S$, et al. Impact of cigarette smoking on the incidence of type 2 diabetes mellitus in middle-aged Japanese men: The Osaka Health Survey. Diabet Med. 1999; 16(11):951-5.

28. Manson JE, Ajani UA, Liu S, Nathan DM, Hennekens $\mathrm{CH}$. A prospective study of cigarette smoking and the incidence of diabetes mellitus among US male physicians. Am J Med. 2000; 109(7):538-42.

Recebido em: 29/7/2008

Versão final reapresentada em: 6/10/2009

Aprovado em: 2/3/2009 